\title{
Exposition radiologique des personnels affectés aux opérations de maintenance de radar de surveillance aérienne
}

\author{
X. MICHEL ${ }^{1}$, D. SCHOULZ ${ }^{2}$, G . ABOU ANOMA ${ }^{3}$, A. CAZOULAT ${ }^{1}$, \\ J.-C. AMABILE ${ }^{1}$, P. LAROCHE ${ }^{1}$
}

(Manuscrit reçu le 27 mars 2012, accepté le 25 juillet 2012)

RÉSUMÉ Le Service de protection radiologique des Armées (SPRA) a mené une étude afin d'évaluer l'exposition des personnels en charge de la maintenance d'un radar de type Palmier sur une base aérienne. Le but de cette étude est d'estimer les doses reçues, de mesurer les différents débits d'équivalents de dose ambiant et de définir un zonage radiologique. Au terme de deux campagnes de mesures, les doses efficaces enregistrées par des dosimètres passifs OSL Inlight ${ }^{\circledR}$ corps entier ont permis de conforter les résultats obtenus par les mesures radiamétriques. Par ailleurs, les débits d'équivalents de dose varient de façon importante en fonction de la position de l'opérateur dans la zone des émetteurs. À partir de ces résultats, les auteurs préconisent des recommandations concernant le zonage radiologique, la catégorisation et les modalités de surveillance dosimétrique des personnels.

ABSTRACT Radiation exposure of workers assigned to the maintenance of air surveillance radar. The French Defence Radiation Protection Service (SPRA) conducted a study to assess the radiation exposure of personnel assigned to the maintenance of the Palmier radar in an Air Force Base. The aim of the study was the assessment of the annual effective doses received by personnel assigned to these maintenance operations, and the measurement of equivalent dose rates in the area in order to realise radiological zoning. In two measurement campaigns, the annual individual effective doses, measured by passive whole-body OSL Inlight ${ }^{\circledR}$ dosimeters, consolidated the results obtained by radiometric measurements. Moreover, the equivalent dose rate shows wide variations in relation to the position of the operator in the emitter's area. From these results, the authors propose recommendations for categorisation of workers, radiological zoning and dose monitoring procedures.

Keywords: radar / klystron / X-ray / radiation exposure / personnel dosimetry

\section{Introduction}

L'Armée de l'Air dispose pour ses besoins opérationnels de différents types de radars, comme le radar multi-lobes Palmier, situés sur des bases aériennes (BA).

\footnotetext{
${ }^{1}$ Service de protection radiologique des armées (SPRA), 1bis rue du Lieutenant Raoul Batany, 92141 Clamart Cedex, France. ${ }^{2}$ Commissariat à l'énergie atomique et aux énergies alternatives, Direction des applications militaires, 91680 Bruyères-leChâtel, France.

${ }^{3}$ Service de médecine de prévention de la Défense, fort neuf de Vincennes, cours des maréchaux, 75614 Paris Cedex 12, France.
} 
Ces radars assurent le guidage des avions et participent à la défense anti-aérienne du territoire national. Si les risques liés à l'exposition aux champs électromagnétiques hyperfréquences émis par ces radars sont aujourd'hui mieux appréhendés, ceux engendrés par l'émission de rayonnements ionisants du fait de leur fonctionnement sont en revanche assez méconnus.

Mis en place dans les années 70, le parc actuel voit sa durée de vie augmenter du fait du glissement progressif des programmes de remplacement. Il apparait donc nécessaire de réévaluer les risques liés à ces installations qui bénéficient d'une surveillance de l'ambiance depuis les années 90. Déjà, en 2008, suite à la mise en évidence d'un dysfonctionnement du système, une vérification du poste avait été menée conduisant à la mise en place d'une surveillance trimestrielle des personnels, pour s'assurer que la dose efficace ne dépassait pas la limite publique (décret 2003-296).

Au ministère de la Défense, la règlementation concernant l'exposition des travailleurs aux risques induits par les rayonnements suit les impératifs de la législation nationale. À ce titre, le chef d'organisme est tenu par une obligation générale de sécurité. Il doit mettre en œuvre toute mesure permettant d'inventorier, d'évaluer et de diminuer l'ensemble des risques, en particulier effectuer les études de postes (IRSN, 2010) en y associant la personne compétente en radioprotection (PCR) de l'établissement.

Dans le cadre de la règlementation propre à la Défense, il fait parti des attributions du Service de protection radiologique des Armées (SPRA, 2005), d'apporter son soutien technique dans le domaine de la radioprotection. Il contribue ainsi à mieux cerner les risques en évaluant l'exposition des personnels affectés aux opérations de maintenance d'un radar Palmier sur une base aérienne et à proposer des mesures pour assurer la pérennité de l'efficacité de ces dispositions.

\section{Rôle du SPRA dans le cadre de la réglementation au ministère de la Défense}

À l'exception des installations nucléaires de base secrètes (qu'elles soient civiles ou militaires) qui relèvent de l'Autorité de sureté du nucléaire de défense (ASND), toutes les autres installations du ministère de la Défense, en tant de paix, sont gérées par la même règlementation que les installations civiles.

Pour pouvoir effectuer ses missions, le SPRA, appui technique du ministère de la Défense, est constitué de deux divisions. D'une part, la division technique, qui comporte un laboratoire de dosimétrie passive, un laboratoire de contrôle radiotoxicologique et un bureau de surveillance radiologique. D'autre part, la division 
médicale, qui est constituée de comporte le bureau de radioprotection médicale, le bureau d'autorisation et d'inventaire des sources et un bureau d'exploitation des données individuelles.

Dans le cadre de cette étude, la totalité des entités a été impliquée permettant d'apporter à l'exploitant du radar une aide méthodologique et technique de haut niveau couvrant tous les domaines de la radioprotection. Par exemple, préalablement à l'étude de poste, une information portant sur les risques des rayonnements ionisants a été effectuée par le médecin du SPRA au profit de la base aérienne.

\section{Chronologie des évènements ayant conduit à l'étude de poste}

Depuis la mise en place de la surveillance radiologique de l'installation (dosimétrie d'ambiance) dans les années 90, d'abord mensuelle puis devenue trimestrielle en 2005, le service de dosimétrie du SPRA envoie, récupère, assure la lecture et exploite les résultats. Jusqu'en juillet 2008, aucune anomalie n' avait été relevée.

Début juillet 2008, le SPRA réceptionne la dosimétrie d'ambiance du second trimestre 2008. Celle-ci est lue dans la semaine suivant le retour et met en évidence une dose anormalement élevée de $146 \mathrm{mSv}$ mesurée au niveau d'un grillage de protection d'un des émetteurs du radar Palmier (Fig. 1). La personne compétente en radioprotection (PCR) est alertée immédiatement par téléphone.

Le 15 juillet 2008, le commandant de la base décide d'arrêter l'émetteur et diligente une enquête technique. Les investigations concluent à une pollution par
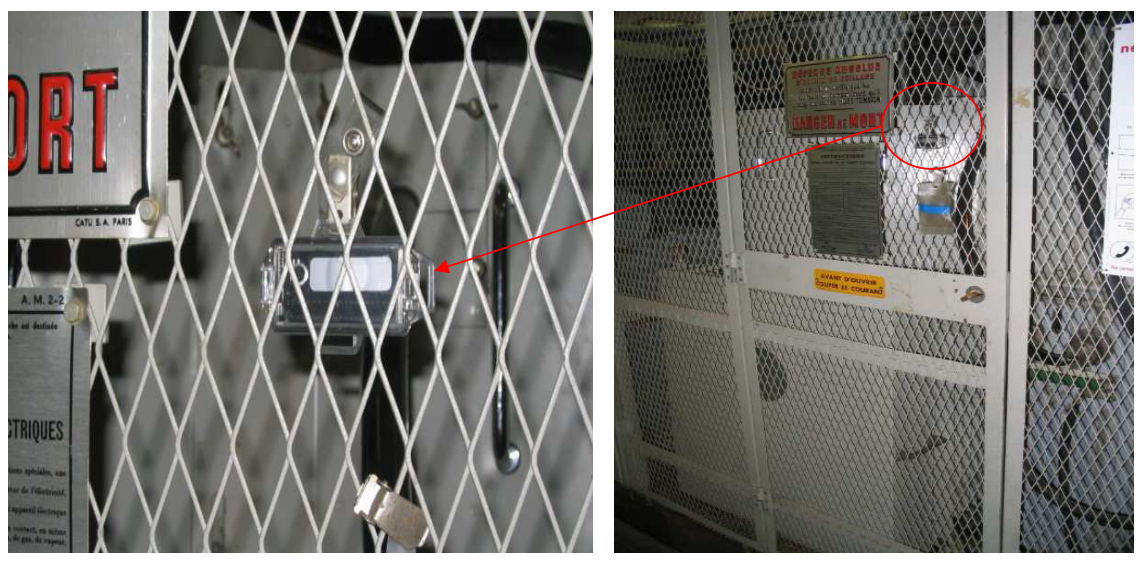

Figure 1 -Dosimètre d'ambiance placé au niveau d'un grillage de protection de l'émetteur du radar Palmier.

Ambient dosimeter placed on the protection gate of the Palmier radar emitter. 


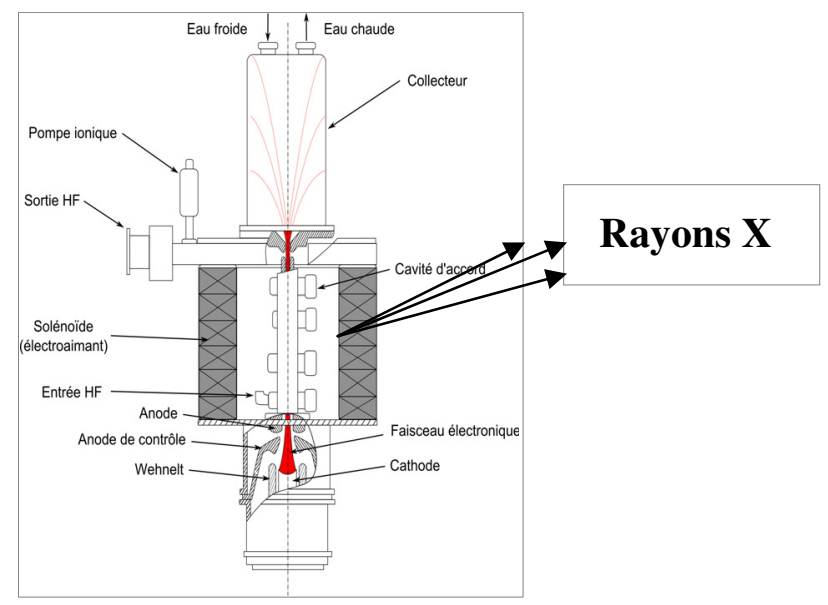

Figure 2 -Défocalisation du faisceau du klystron et émission d'un rayonnement X de freinage.

Defocusing of klystron beam and emission of bremsstrahlung $X$-rays.

l'huile des compresseurs qui a entraîné une désadaptation du tube électronique de forte puissance (klystron) avec le guide d'onde de sortie. En conséquence, une partie de l'onde électromagnétique émise par ce tube est venue frapper par défocalisation du faisceau, les structures métalliques avoisinantes générant ainsi, outre un échauffement important, une importante émission X (Fig. 2).

Le 5 août 2008, l'équipe technique de l'Armée de l'Air chargée de la surveillance et de la maintenance de ces radars, effectue des mesures complémentaires autour de l'émetteur en fonctionnement avec un radiamètre de type Babyline ${ }^{\circledR}$. Selon l'emplacement, les résultats de mesure montrent des valeurs de $10 \mu \mathrm{Sv} / \mathrm{h}$ et $2 \mathrm{mSv} / \mathrm{h}$ pour un bruit de fond de l'environnement 0,03 à $0,13 \mu \mathrm{Sv} / \mathrm{h}$.

Le 14 août 2008, le tube klystron est remplacé et le matériel défaillant est envoyé à l'industriel pour expertise. La PCR assure les mesures de réception qui sont normales.

Le 23 décembre 2008, une société de contrôle externe procède à des mesures de vérification, effectuées avec une Babyline ${ }^{\circledR}$, qui ne mettent plus en évidence d'émission X parasites.

Le commandant de la base aérienne décide alors de remettre l'émetteur en fonctionnement le 21 janvier 2009, date de réception du rapport du contrôle externe. Cette décision est assortie d'un certain nombre de mesures dont le port d'une dosimétrie opérationnelle avant toute intervention dans la salle des émetteurs. 
Dans ce contexte, la structure intégrée du maintien en condition opérationnelle des matériels aéronautique de la Défense (SIMMAD) demande alors au SPRA la réalisation d'une campagne de mesures approfondie sur les radars sol/air en service dans les armées, et en particulier, sur les radars multilobes Palmier sur le site d'une base aérienne.

Une première étude dosimétrique, menée de mai à décembre 2009 a pour objectif :

- de réaliser une cartographie dosimétrique d'ambiance de l'installation ;

- de définir le zonage radiologique dans la salle des émetteurs du radar Palmier lors des opérations de maintenance ;

- d'évaluer les doses efficaces annuelles potentiellement reçues par les personnels affectés à ces opérations de maintenance.

L'indisponibilité technique de l'un des deux émetteurs lors des mesures de décembre 2009 ne permet pas de réaliser la totalité des mesures au cours d'une opération de maintenance. Il a été décidé de compléter l'étude lorsque les deux émetteurs auront retrouvé leur disponibilité.

Compte tenu des impératifs opérationnels de l'installation, les mesures complémentaires n'ont pu être effectuées qu'à partir de janvier 2012.

\section{Caractéristiques de l'installation}

Le radar Palmier (Fig. 3) est un système de détection de la défense aérienne de l'Armée de l'Air capable d'émettre une impulsion électromagnétique maximale oscillant entre 14 et 20 mégawatts (MW) pour une tension appliquée de 20 à 30 kilovolts $(\mathrm{kV})$.

Son fonctionnement est relativement simple. Un émetteur envoie des ondes radio qui, réfléchies sur une cible, sont alors détectées par un récepteur situé le plus souvent au même endroit que l'émetteur. La position de la cible est estimée grâce au retour du signal, sa vitesse est mesurée par effet doppler à partir du changement de fréquence de ce signal.

Le système du radar Palmier possède deux émetteurs de champs électromagnétiques indépendants, fonctionnant de manière simultanée, mais seul un des deux émetteurs peut être utilisé pour la détection radar. En effet, un des deux émetteurs, dit «émetteur radar » est orienté vers l'espace aérien pendant que le deuxième émetteur, dit « émetteur charge » est orienté en direction d'une cible fixe nommé «charge » qui permet la dissipation de l'énergie sous forme de chaleur. Un commutateur permet la bascule d'un émetteur à l'autre. Ainsi, l'émetteur radar en fonctionnement est toujours à une puissance plus importante que l'émetteur charge. 

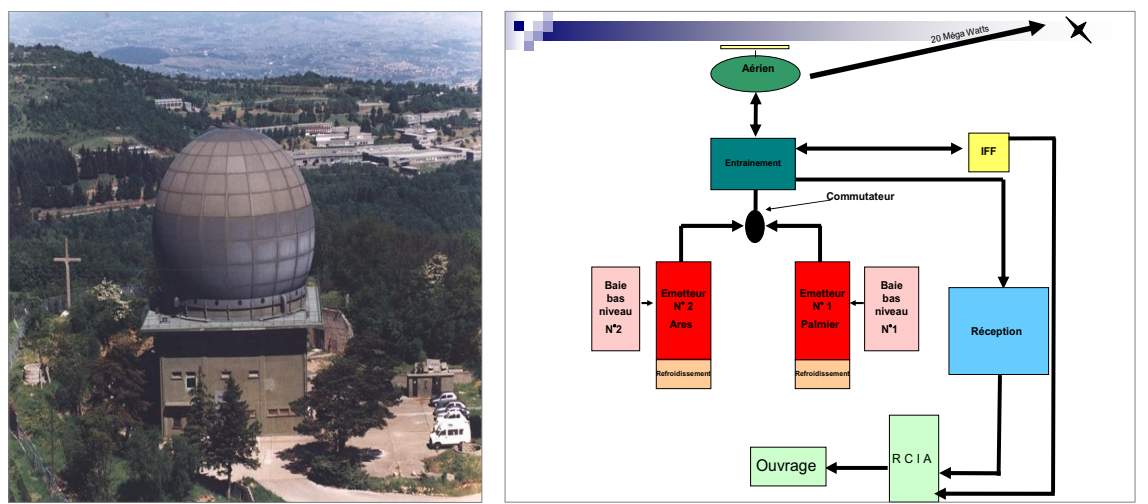

Figure 3 - Radar Palmier et tableau synoptique de fonctionnement.

Palmier radar and system table.

Chaque émetteur est constitué de deux chaînes d'amplification :

- une baie de bas niveau de puissance ;

- une baie de haut niveau de puissance.

La baie de bas niveau de puissance génère un signal dont les fréquences sont comprises dans la bande $\mathrm{S}$ entre 2 et 4 GigaHertz $(\mathrm{GHz})$. Cette impulsion est envoyée à un émetteur de haut niveau de puissance.

La baie de haut niveau de puissance n'intervient ni sur la fréquence ni sur la forme de l'impulsion mais uniquement sur la puissance du signal de sortie, qui passe de $2 \mathrm{~kW}$ à $20 \mathrm{MW}$. Ce gain de puissance fait appel à l'utilisation d'un tube électronique de forte puissance appelé klystron (Fig. 4).

Le signal haute fréquence en entrée du klystron est amplifié grâce à un faisceau d'électrons produit par un filament chauffé. Ce faisceau électronique est modulé en amplitude en traversant une première cavité résonnante excitée par le signal hyperfréquence. L'énergie est ensuite amplifiée dans les cavités résonnantes suivantes jusqu'à la cavité de sortie. Dans cette dernière cavité, les électrons cèdent partiellement leur énergie cinétique sous forme d'un rayonnement électromagnétique qui est alors récupéré grâce à un circuit d'accord relié à la cavité. L'onde électromagnétique émise par le tube permet de canaliser la quasitotalité de l'énergie vers l'antenne via les guides d'onde pressurisés.

Dans les klystrons, modulant ou amplifiant les émissions hyperfréquences, les électrons qui ne sont pas canalisés produisent par rayonnement de freinage des rayonnements $\mathrm{X}$ parasites. 


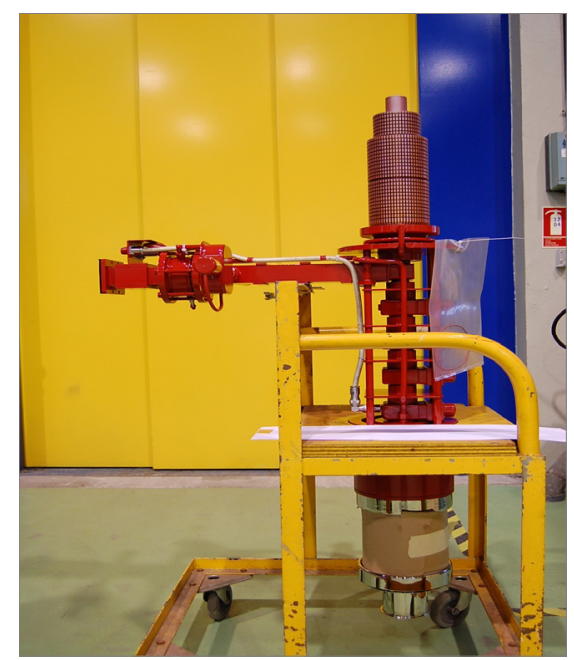

Figure 4-Le klystron, tube électronique de forte puissance.

The klystron, a high-power electronic tube.

Ce spectre d'énergie $\mathrm{X}$ ne dépasse pas une trentaine de $\mathrm{keV}$ au maximum compte tenu de la tension d'alimentation appliquée en moyenne de 20 à $30 \mathrm{kV}$. Une spectrométrie réalisée à l'aide d'un Inspector $2000^{\circledR}$ a permis de confirmer cette valeur.

En conditions opérationnelles, un émetteur ne peut pas fonctionner plus d'une heure. Si cette durée est atteinte, le commutateur bascule automatiquement sur l'autre émetteur. C'est donc la configuration la plus pénalisante - une heure de fonctionnement continu pour l'émetteur le plus puissant - qui a été retenue pour l'étude de poste.

\section{Analyse des tâches}

L'étude des postes de travail a nécessité au préalable l'analyse de la tâche, c'est-àdire, la définition d'un parcours type (Fig. 5) et l'estimation des temps nécessaires à l'accomplissement des différentes actions pendant une opération « normale » de maintenance dans la salle des émetteurs du radar Palmier.

L'émetteur numéro 2, permettant d'atteindre une plus grande puissance (16,5 MW) que l'émetteur numéro 1 (14 MW), a été choisi comme «émetteur radar » tandis que l'émetteur numéro 1 était considéré comme «émetteur charge ».

Après échange avec l'équipe de prévention de la base aérienne, ces données ont été analysées. Les résultats présentés dans le tableau I représentent la moyenne des 


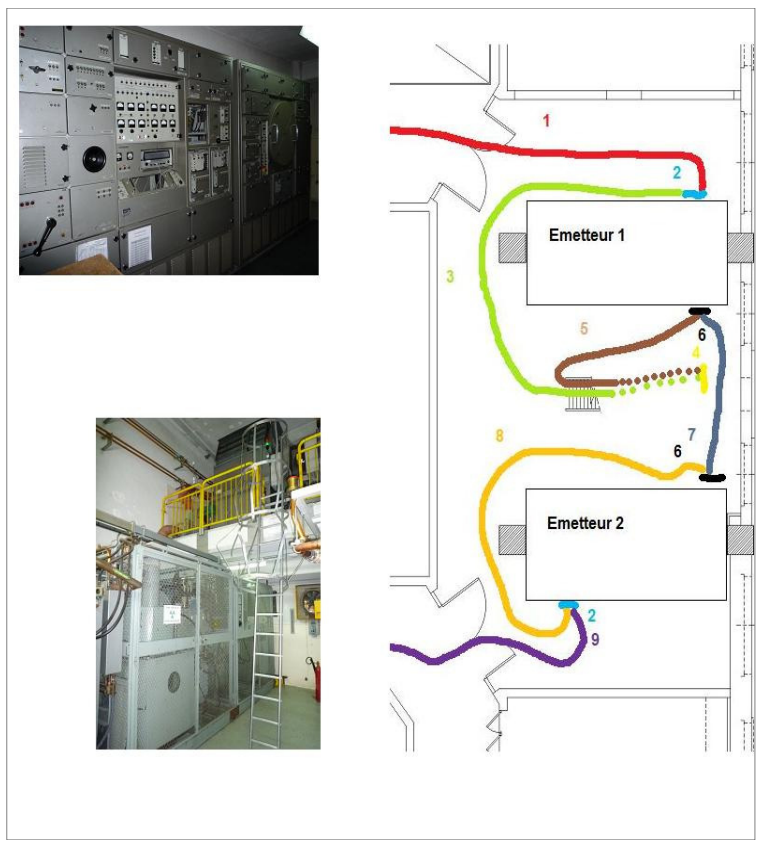

Figure 5 - Parcours type d'un opérateur lors d'une opération de maintenance.

Type of maintenance round done by an operator.

temps passés pour les différents travaux par l'ensemble des équipes de maintenance, soit 14 personnes.

Ce parcours type est effectué en moyenne quatre fois par semaine. Ce budget espace temps a été retenu pour l'expression des résultats dans la suite de cet article. Pour la mise en place ultérieure du zonage, un coefficient de majoration sera utilisé pour raisons de sécurité.

\section{Matériels et méthodes}

\subsection{Matériels}

La mesure radiamétrique en ambiance électromagnétique peut être fortement perturbée. L'appareillage électronique doit donc être adapté et bénéficier de la norme comptabilité électromagnétique ${ }^{4}$.

${ }^{4}$ Norme NF-EN 61000-4-1, Compatibilité électromagnétique (CEM). Partie 4-1: Techniques d'essai et de mesure. 
TABLEAU I

Estimation des temps des différentes actions lors d'une maintenance. Time estimation of actions during a maintenance operation.

\begin{tabular}{cll}
\hline $\mathrm{N}^{\circ}$ & \multicolumn{1}{c}{ Action } & \multicolumn{1}{c}{ Durée } \\
\hline 1 & Entrée dans la salle des émetteurs (P1) & 10 secondes \\
\hline 2 & Mesures journalières à l'oscilloscope (FS12) & 10 minutes (émetteur en marche) \\
\hline 3 & $\begin{array}{l}\text { Montée aux aérocondenseurs et vérification les débits } \\
\text { d'eau du système de refroidissement (FS7) }\end{array}$ & 30 secondes \\
\hline 4 & Prise des températures (FS7) & 3 minutes \\
\hline 5 & Redescente (FS7) & 30 secondes \\
\hline 6 & Mesures sur les parties énergie des émetteurs (FS8) & 1 minute \\
\hline 7 & Changement d'émetteur & 2 secondes \\
\hline 6 & Mesures sur les parties énergie des émetteurs (FS5) & 1 minute \\
\hline 8 & $\begin{array}{c}\text { Aller vers la partie commande de l'autre émetteur et } \\
\text { vérification les débits d'eau du système de refroidissement } \\
\text { (FS3) }\end{array}$ & 10 secondes \\
\hline 2 & Mesures journalières à l'oscilloscope (FS2) & 3 minutes (émetteur de veille) \\
\hline 9 & Sortie de la salle des émetteurs (P2) & 10 secondes \\
\hline
\end{tabular}

D'autre part, les détecteurs (radiamètres, dosimètres) doivent avoir une réponse en énergie compatible avec le spectre X émis et une sensibilité suffisante compte tenu des faibles niveaux attendus.

Les caractéristiques des différents détecteurs utilisés seront présentées succinctement dans les différents paragraphes de la méthodologie ci après.

Les différentes grandeurs mesurées seront l'équivalent de dose individuelle $H p(10)$ pour les estimations aux postes de travail et l'équivalent de dose ambiant $H^{*}(10)$ pour les mesures d'ambiance conduisant à la cartographie et au zonage de l'installation.

En comparaison avec les mesures radiamétriques, des dosimètres OSL ont été mis en place comme dosimètres d'ambiance sans fantôme. Un facteur de correction a été pris en compte dans leur interprétation.

\subsection{Méthodes}

\subsubsection{Cartographie dosimétrique d'ambiance de l'installation}

La cartographie dosimétrique d'ambiance a été réalisée au moyen de dosimètres passifs OSL InLight ${ }^{\circledR}$, sensibles aux rayonnements $\gamma$ et X de $5 \mathrm{keV}$ à $40 \mathrm{MeV}$, avec 


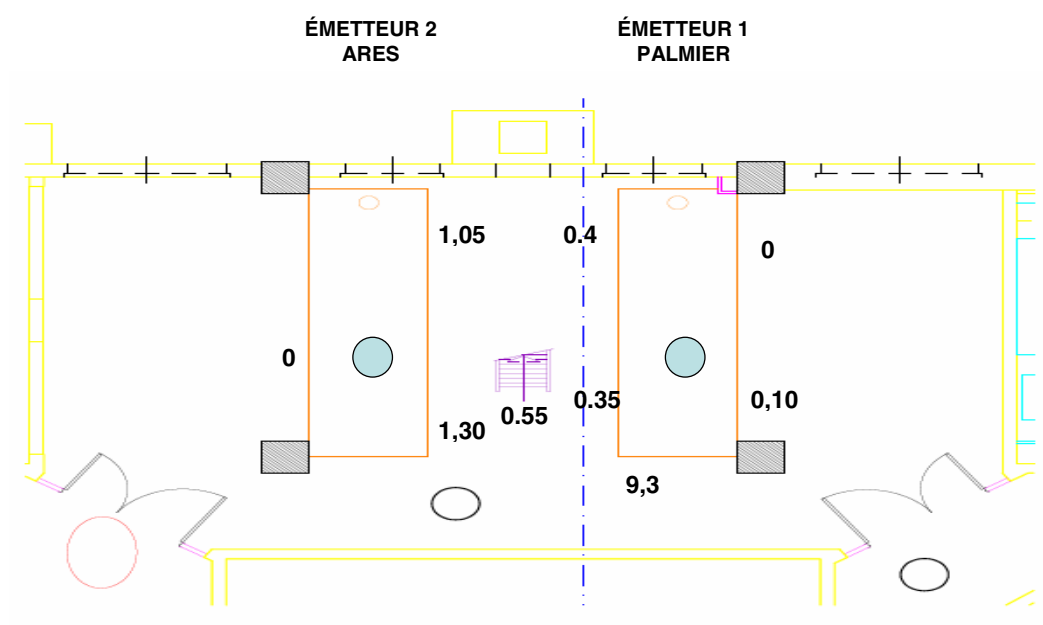

Figure 6 - Plan de la salle des émetteurs du radar Palmier avec les résultats dosimétriques en mSv par mois.

Palmier radar emitter room map with dosimetric results.

un seuil de détection de $0,10 \mathrm{mSv}$. Neuf dosimètres OSL ont été placés dans différents points de l'installation le 27 mai 2009 (Fig. 6). Ces dosimètres ont enregistré sur une période d'un mois (juin 2009) l'équivalent de dose individuel $H p(10)$ au niveau de chaque matériel et voie de circulation dans le local des émetteurs.

\subsubsection{Radiamétrie}

En vue de réaliser le zonage radiologique et conformément à l'arrêté du 15 mai 2006 (JORF, 2006), l'équivalent de dose ambiant est mesuré par radiamétrie dans les conditions les plus pénalisantes. Celles-ci correspondent à un fonctionnement continu d'une heure. Il n'a donc pas été possible de réitérer cette opération compte tenu de l'occupation opérationnelle du radar. L'estimation des erreurs de mesure a donc été effectuée en tenant compte des incertitudes fournies par le constructeur pour l'AT1123 ${ }^{\circledR}$ (réponse en énergie au ${ }^{137} \mathrm{Cs} \pm 35 \%$ de $15 \mathrm{keV}$ à $60 \mathrm{keV}$ ) et par le laboratoire de dosimétrie du SPRA (incertitude globale $40 \%$ de $5 \mathrm{keV}$ à $40 \mathrm{MeV}$ ) pour les valeurs fournies par les dosimètres individuels ou d'ambiance.

Une comparaison est effectuée avec des dosimètres passifs placés aux points de mesure radiamétriques et ayant ont intégré la dose pendant une heure (Fig. 7).

Les mesures ont été réalisées à l' aide deux radiamètres AT1123 ${ }^{\circledR}$ en des points choisis par référence au parcours type d'une ronde de maintenance. L'AT1123 ${ }^{\circledR}$ est 


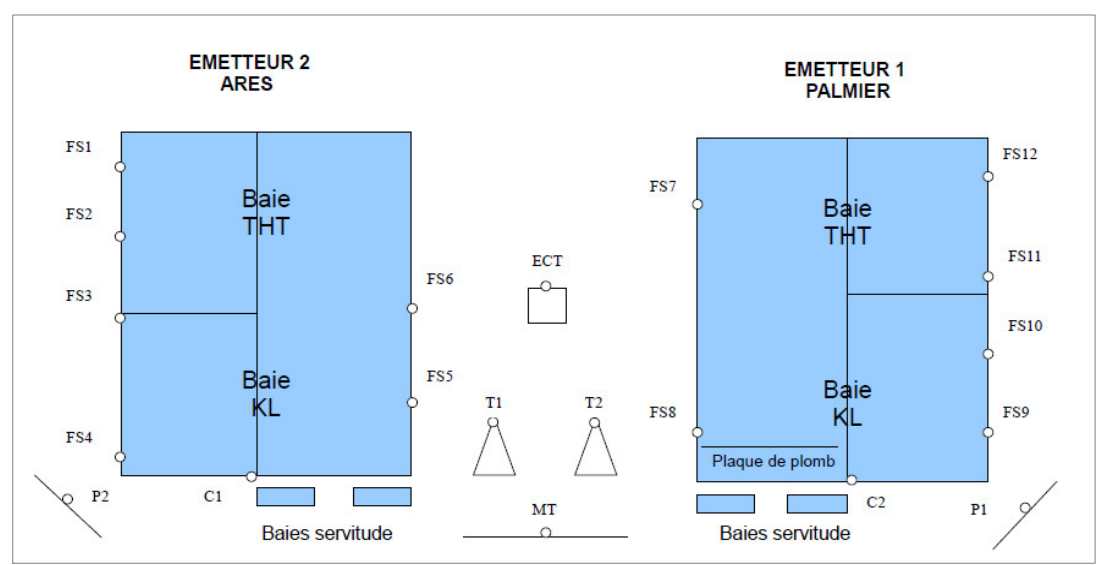

Figure 7 - Plan de la salle des émetteurs du radar Palmier avec les emplacements des dosimétres OSL pour le zonage radiologique.

Palmier radar emitter room map with location of OSL dosimeters for radiological zonage.

un détecteur à scintillation qui couvre une gamme d'énergie de $15 \mathrm{keV}$ à $10 \mathrm{MeV}$, pour des débits d'équivalent de dose ambiant de $50 \mathrm{nSv} / \mathrm{h}$ à $10 \mathrm{~Sv} / \mathrm{h}$. Il bénéficie de la norme compatibilité électromagnétique.

Deux types de mesures ont été réalisés :

- mesures de doses intégrées sur 5 minutes rapportées à une heure ;

- mesures de débits de doses instantanés en $\mu \mathrm{Sv} / \mathrm{h}$.

\subsection{Doses efficaces individuelles reçues lors des opérations de maintenance}

Pour l'évaluation de la dose efficace reçue, tous les personnels affectés aux opérations de maintenance dans la salle des émetteurs du radar Palmier ont porté pendant trois mois (de décembre 2009 à février 2010), au niveau de la poitrine, un dosimètre passif OSL InLight ${ }^{\circledR}$.

\section{Résultats}

Ils expriment les trois types de mesures réalisées.

\subsection{Mesures dans la salle des émetteurs}

Ils sont rapportés dans le tableau II et la figure 7. Ils présentent la dose intégrée $(\mathrm{mSv})$ par les OSL InLight ${ }^{\circledR}$ sur une heure, les réponses du radiamètre AT1123 ${ }^{\circledR}$ 
en intégration $(\mu \mathrm{Sv})$ sur 5 minutes (régime pulsé du radar), ces mêmes mesures extrapolées à une heure $(\mu \mathrm{Sv} / \mathrm{h})$, et les mesures toujours avec AT1123 ${ }^{\circledR}$ en instantané $(\mu \mathrm{Sv} / \mathrm{h})$. Ces dernières correspondent à des mesures crêtes.

TABLEAU II

Mesures des doses et débits de dose intégrés lors d'un parcours type de maintenance $\left(\mathrm{OSL}_{\text {, AT1123 }}^{\circledR}\right)$.

Doses and dose rates during a typical maintenance round (OSL, AT1123 ${ }^{\circledR}$ ).

\begin{tabular}{cccc}
\hline $\begin{array}{c}\text { Point de } \\
\text { mesure }\end{array}$ & $\begin{array}{c}\text { OSL sur 1 heure } \\
(\mathbf{m S v})\end{array}$ & $\begin{array}{c}\text { AT1123 sur } \mathbf{5} \text { min } \rightarrow \mathbf{1} \text { heure } \\
(\boldsymbol{\mu S v})\end{array}$ & $\begin{array}{c}\text { AT1123 en instantané } \\
(\boldsymbol{\mu S \mathbf { S }} / \mathbf{h})\end{array}$ \\
\hline FS1 & 0,03 & $0,061 \rightarrow 0,732$ & 0,30 \\
FS2 & 0,03 & $0,043 \rightarrow 0,516$ & 4,90 \\
FS3 & 0,02 & $0,058 \rightarrow 0,696$ & 2,30 \\
FS4 & 0 & $0,034 \rightarrow 0,408$ & 0,70 \\
FS5 & $\mathbf{0 , 1 1}$ & $0,156 \rightarrow 1,872$ & $\mathbf{2 7 , 9 0}$ \\
FS6 & 0,02 & $0,124 \rightarrow 1,488$ & $\mathbf{2 6 , 4 5}$ \\
FS7 & 0,03 & $0,044 \rightarrow 0,528$ & $\mathbf{2 3}$ \\
\hline FS8 & 0,02 & $0,048 \rightarrow 0,576$ & $\mathbf{2 5 , 6 0}$ \\
FS9 & 0,01 & $0,105 \rightarrow 1,26$ & 0,20 \\
\hline FS10 & 0,01 & $0,078 \rightarrow 0,936$ & 0,20 \\
\hline
\end{tabular}

\begin{tabular}{cccc}
\hline $\begin{array}{c}\text { Point de } \\
\text { mesure }\end{array}$ & $\begin{array}{c}\text { OSL sur 1 heure } \\
(\mathbf{m S v})\end{array}$ & $\begin{array}{c}\text { AT1123 } \mathbf{s u r} \mathbf{5} \text { } \mathbf{\text { in}} \rightarrow \mathbf{1 h e u r e} \\
(\boldsymbol{\mu S v})\end{array}$ & $\begin{array}{c}\text { AT1123 en instantané } \\
(\boldsymbol{\mu S v} \mathbf{h})\end{array}$ \\
\hline FS11 & 0,04 & $0,023 \rightarrow 0,276$ & 0,35 \\
FS12 & 0 & $0,025 \rightarrow 0,300$ & 0,20 \\
C1 & 0,01 & $0,043 \rightarrow 0,516$ & 4,80 \\
C2 & 0,01 & $0,058 \rightarrow 0,696$ & 0,60 \\
P1 & 0,02 & $0,025 \rightarrow 0,300$ & 0,35 \\
P2 & 0,04 & $0,029 \rightarrow 0,348$ & 0,20 \\
ECT & 0,01 & $0,090 \rightarrow 1,080$ & 11,20 \\
MT & 0,02 & $0,060 \rightarrow 0,720$ & 13,60 \\
T1 & 0,03 & $0,058 \rightarrow 0,696$ & 17 \\
T2 & 0,02 & $0,121 \rightarrow 1,452$ & 8,80 \\
\hline
\end{tabular}

La dose instantanée maximale totale mesurée de 27,9 $\mu \mathrm{Sv} / \mathrm{h}\left(\mathrm{AT} 1123^{\circledR}\right.$ ) est considérée comme la plus pénalisante, les émetteurs étant dans les configurations les plus défavorables pour les intervenants. L'essentielle de cette dose est enregistrée au niveau des portes grillagées du compartiment des émetteurs. L'ambiance à l'intérieur des compartiments grillagés n'a pas été mesurée car ils ne 
TABLEAU III

Résultats dosimétriques d'ambiance (juin 2009). Ambient dosimetric results (June 2009).

\begin{tabular}{|c|c|c|c|c|c|}
\hline Intitulé & Position & Date début & Date fin & Type & Dose en mSv \\
\hline Témoin & $\mathrm{T}$ & $01 / 06 / 2009$ & $30 / 06 / 2009$ & $\mathrm{G}-\mathrm{X}$ & $\mathbf{0 , 0 0}$ \\
\hline $\begin{array}{l}\text { Entre baie THT et baie klystron } \\
\text { émetteur } 1 \text { (FS 10) }\end{array}$ & $\mathrm{Z}$ & 01/06/2009 & $30 / 06 / 2009$ & $\mathrm{G}-\mathrm{X}$ & 0,10 \\
\hline Baie eau émetteur 1 (FS12) & $\mathrm{Z}$ & 01/06/2009 & $30 / 06 / 2009$ & $\mathrm{G}-\mathrm{X}$ & $\mathbf{0 , 0 0}$ \\
\hline Plombage klystron émetteur 1 (FS8) & $\mathrm{Z}$ & 01/06/2009 & $30 / 06 / 2009$ & $\mathrm{G}-\mathrm{X}$ & 0,35 \\
\hline Arrivée baie bas niveau émetteur 1 (C2) & $\mathrm{Z}$ & 01/06/2009 & $30 / 06 / 2009$ & $\mathrm{G}-\mathrm{X}$ & 9,30 \\
\hline Passerelle émetteur 1 (FS7) & $\mathrm{Z}$ & 01/06/2009 & $30 / 06 / 2009$ & G-X & $\mathbf{0 , 4 0}$ \\
\hline Cable LAR émetteur 1 (ECT) & $\mathrm{Z}$ & 01/06/2009 & $30 / 06 / 2009$ & G-X & 0,55 \\
\hline $\begin{array}{l}\text { Entre baie THT et baie klystron } \\
\text { émetteur } 2 \text { (FS3) }\end{array}$ & $\mathrm{Z}$ & 01/06/2009 & $30 / 06 / 2009$ & $\mathrm{G}-\mathrm{X}$ & $\mathbf{0 , 0 0}$ \\
\hline Plombage klystron émetteur 2 (FS5) & $\mathrm{Z}$ & 01/06/2009 & $30 / 06 / 2009$ & G-X & 1,30 \\
\hline Passerelle émetteur 2 (FS6) & $\mathrm{Z}$ & 01/06/2009 & $30 / 06 / 2009$ & $\mathrm{G}-\mathrm{X}$ & 1,05 \\
\hline
\end{tabular}

$\mathrm{Z}$ : ambiance ; $\mathrm{T}$ : Témoin ; G-X : dosimètres permettant la mesure gamma-X.

sont pas, en pratique, accessibles lors d'une ronde classique de maintenance (existence d'un grillage de protection).

\subsection{Dosimétrie d'ambiance de l'installation}

Les résultats dosimétriques d'ambiance mesurés $(\mathrm{mSv})$ et rapportés au plan du local sont donnés dans le tableau III et la figure 6 .

Les résultats d'ambiance mettent en évidence l'existence de deux points particuliers au niveau des grillages face aux klystrons :

- émetteur numéro $1=9,3 \mathrm{mSv}$ (sur 1 mois) ;

- émetteur numéro $2=1,3 \mathrm{mSv}$ (sur 1 mois).

\subsection{Doses efficaces individuelles reçues}

Le rapport d'essai édité par le laboratoire de dosimétrie est reproduit au tableau IV. Parmi les personnels de maintenance : huit résultats n'ont pas dépassé le seuil de détection du dosimètre, cinq ont reçu une dose efficace de $0,10 \mathrm{mSv}$ et une dose efficace maximale de $0,25 \mathrm{mSv}$ a été enregistrée sur trois mois. 
TABLEAU IV

Résultats de la dosimétrie individuelle passive trimestrielle corps entier. Results of trimestrial individual dosimetry $(\mathbf{E})$.

\begin{tabular}{|c|c|c|c|c|c|c|c|c|c|}
\hline \multicolumn{2}{|l|}{ Rapport d'essais $n^{\circ} 51525$} & \multicolumn{5}{|c|}{ 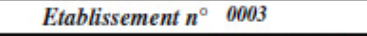 } & \multirow[b]{2}{*}{ Eiat } & \multicolumn{2}{|r|}{$12 / 2009$} \\
\hline 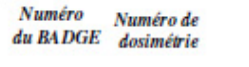 & libellé & $\begin{array}{c}\text { Code } \\
\text { Emploi }\end{array}$ & Type & Position & $\begin{array}{c}\mathrm{Hp} 10 \\
\text { en } \mathrm{mSv}\end{array}$ & $\begin{array}{l}\mathrm{Hp0,07} \\
\text { en } \mathrm{mSv}\end{array}$ & & $\begin{array}{c}\text { Cumul } \\
\text { Hp10 } \\
\text { en } m S v\end{array}$ & $\begin{array}{l}12 \text { mois } \\
\text { Hp Q,07 } \\
\text { en } \mathrm{mSv}\end{array}$ \\
\hline & & Libellé $d t$ & $u G R C$ & UPE : & Occ & casionnel & & & \\
\hline XA01256196C & & & G.X & $\begin{array}{ll}T \\
T\end{array}$ & 0,00 & 0,00 & & & \\
\hline XA01146352N 070724 & & 399 & G.X & $O E$ & 0,00 & 0,00 & & 0,000 & 0,000 \\
\hline XA01146363K 182714 & & 399 & $G-X$ & $O E$ & 0,10 & 0,15 & & 0,100 & 0,150 \\
\hline XA011378467 182715 & & 399 & G.X & $O E$ & 0,00 & 0,10 & & 0,000 & 0,100 \\
\hline XA01145364J $182 Z 16$ & & 399 & 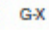 & $O E$ & 0,00 & 0,00 & & 0,000 & 0,000 \\
\hline XA01145890E 182717 & & 399 & 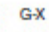 & $O E$ & 0,25 & 0,35 & & 0,250 & 0,350 \\
\hline XA01432055Q $182 / 18$ & & 399 & $G-x$ & $O E$ & 0,10 & 0,15 & & 0,100 & 0,150 \\
\hline XА01432309H 182$] 19$ & & 399 & $G \times x$ & OE & 0,10 & 0,10 & & 0,100 & 0,100 \\
\hline XA01434565D 180991 & & 399 & $G-x$ & $O E$ & 0,10 & 0,10 & & 0,100 & 0,250 \\
\hline XA011466874 182720 & & 399 & $\mathrm{G}-\mathrm{X}$ & $O E$ & 0,00 & 0,00 & & 0,000 & 0,000 \\
\hline XA011467781 182721 & & 399 & G.X & $O E$ & 0,00 & 0,00 & & 0,000 & 0,000 \\
\hline XA01432438E $\quad 182722$ & & 399 & $G-x$ & $O E$ & 0,00 & 0,00 & & 0,000 & 0,000 \\
\hline XA01144717B 182723 & & 399 & G.X & $\mathrm{OE}$ & 0,10 & 0,10 & & 0,100 & 0,100 \\
\hline XA01145198C 182724 & & 399 & $G-x$ & $O E$ & 0,00 & 0,00 & & 0,000 & 0,000 \\
\hline XA01434707B 182725 & & 399 & $G-x$ & $O E$ & 0,00 & 0,00 & & 0,000 & 0,000 \\
\hline
\end{tabular}

\section{Discussion}

Les résultats de cette étude dosimétrique permettent de formuler des recommandations concernant le zonage radiologique, la catégorisation des travailleurs, les mesures d'optimisation de la radioprotection et le contrôle radiologique. Le régime d'autorisation de l'installation sera également abordé.

\subsection{Zonage radiologique}

Les résultats rapportés dans le tableau II ont permis de le déterminer en les comparant avec les limites réglementaires (ASN, 2008).

Il faut noter que les OSL InLight ${ }^{\circledR}$ ne sont pas adaptés pour réaliser un zonage dans ce type d'installation parce que le seuil de détection est trop bas $(S D=$ $0,10 \mathrm{mSv}$ ) si la mesure est faite sur 1 heure (temps de fonctionnement maximal en continu des radars). Les radars ne pouvant pas fonctionner en mode opérationnel 
sur un temps plus long, nous considèrerons que le temps de fonctionnement représentant les conditions les plus défavorables est de 1 heure.

Les caractéristiques d'émission de l'installation se rapprochent plus d'un champ pulsé que d'un champ continu. De ce fait, l'AT1123 ${ }^{\circledR}$ doit être réglé pour une mesure en champ pulsé pour la détection des rayonnements $\mathrm{X}$ « parasites ». De plus, le temps d'intégration devrait se faire sur des durées longues car des mesures avec l'AT1123 en débit de dose sur 5 minutes semblent sous estimer les doses reçues par rapport aux débits de doses instantanées et à la dosimétrie passive OSL InLight ${ }^{\circledR}$.

Les résultats des mesures réalisées avec l'AT1123 ${ }^{\circledR}$ autour de l'émetteur numéro 2 sont cohérents avec ceux fournis par les dosimètres d'ambiance précisément sur cet émetteur numéro 2 ( $c f$. Tab. III).

À partir des mesures intégrées sur 5 minutes extrapolées à une heure, tous les points de mesures réalisées en dehors des enceintes grillagées de la salle des émetteurs montrent une dose intégrée sur une heure inférieure à 7,5 $\mu \mathrm{Sv}$. Si l'on prend en compte les mesures instantanées, les points de mesures réalisées entre les deux émetteurs, au niveau des grillages d'enceintes (FS5, FS6, FS7 et FS8 ; $c f$. Fig. 7 et Tab. II) révèlent des débits de dose supérieures à $25 \mu \mathrm{Sv} / \mathrm{h}$.

Très logiquement à partir des doses intégrées, le classement de la pièce de circulation devrait être une zone surveillée, la zone contrôlée simple (trisecteur vert) se trouvant au-delà du grillage. Cependant, compte tenu des valeurs crêtes mesurées corroborées par les résultats dosimétriques d'ambiance $(9,3 \mathrm{mSv}$ sur un mois) rapportés dans le tableau III, il a été décidé pendant la période probatoire de surveillance de 3 ans de surévaluer le zonage. Donc la zone de circulation a été classée zone contrôlée simple et il a été crée une zone contrôlée spécialement règlementée signalée par un trisecteur de couleur jaune au delà du grillage, cette zone restant cependant inaccessible.

\subsection{Catégorisation des personnels}

Les résultats de la dosimétrie individuelle passive enregistrés sur une période de trois mois montrent que les doses individuelles susceptibles d'être reçues lors des opérations de maintenance sur le radar Palmier sont comprises entre 0,40 et $1 \mathrm{mSv}$ sur un an (calcul fait à partir des séquences de l'étude des tâches). Ceci est corroboré par la surveillance dosimétrique trimestrielle des personnels (Tab. IV). L'essentiel des doses se situe plutôt dans la fourchette basse (13 doses sur 14 inférieures ou égales à $0,40 \mathrm{mSv} / \mathrm{an}$ ) mais avec un personnel susceptible de 
recevoir une dose efficace maximale annuelle de $1 \mathrm{mSv}$, dose égale à la valeur limite publique réglementaire.

Devant ces résultats « frontières », compte-tenu des incertitudes de mesures et en prenant en compte la circulaire DGT/ASN n ${ }^{\circ} 4$ du 21 avril 2010 (ASN, 2010), le SPRA préconise de classer ces personnels comme travailleurs exposés ${ }^{5}$ de catégorie $\mathrm{B}$ pendant une période probatoire d'un à trois ans. Dans cette éventualité, ces personnels doivent alors bénéficier d'une surveillance médicale renforcée et d'une surveillance dosimétrique individuelle passive trimestrielle.

Les résultats dosimétriques enregistrés sur une période plus longue permettront de confirmer ou éventuellement de lever cette catégorisation.

Compte tenu du zonage préconisé, ces personnels devront posséder une dosimétrie passive et active par dosimètre opérationnel. Il sera nécessaire de choisir un dosimètre opérationnel durci.

\subsection{Optimisation de la radioprotection}

La cartographie dosimétrique d'ambiance en 2009 a identifié deux «points chauds » (débits élevés) face à la sortie des klystrons des émetteurs 1 et 2, au niveau du circuit de refroidissement. L'étude radiamétrique a mis en évidence l'existence d'un autre «point chaud» situé au niveau de l'échangeur à contre courant de l'émetteur 2. La mise en place d'une protection physique par une plaque de protection en plomb a été réalisée afin de limiter l'exposition du personnel à ce niveau.

Ces lieux devront être clairement identifiés et signalisés par la PCR afin d'avertir les personnels du risque d'irradiation et d'y interdire le stationnement.

Des mesures de sécurisation des grilles des émetteurs avec un système d'arrêt de l'alimentation en cas d'ouverture ont également été recommandées.

Ces préconisations ont été effectivement réalisées. Elles ont été prises en compte lors de la définition du zonage correspondant suite aux contrôles de janvier 2012.

\subsection{Contrôle radiologique et régime d'autorisation de l'installation}

Les installations émettrices de rayonnements ionisants doivent faire l'objet d'un contrôle périodique conformément à l'arrêté du 21 mai 2010 (JORF, 2010).

\footnotetext{
5 Est considéré comme travailleur exposé tout travailleur susceptible de dépasser, dans le cadre de son activité professionnelle, l'une des valeurs limites de dose fixées pour le public, quelles que soient les conditions de réalisation de l'opération ; habituelles ou bien liées à un incident.
} 
Pour les contrôles périodiques internes, le SPRA recommande pour la mesure des doses et des débits de dose des rayonnements $\mathrm{X}$ parasites l'utilisation d'un radiamètre type $\mathrm{AT} 1123^{\circledR}$ en remplacement de l'appareil Radeye G20-10 ${ }^{\circledR}$, actuellement utilisé par la PCR du site. En effet, l'AT1123 est adapté à l'émission des rayonnements $\mathrm{X}$ en champs pulsés émis par les tubes klystron, ce qui n'est pas le cas de l'instrumentation actuelle.

À notre connaissance, il n'existe pas de régime d'autorisation de ce type d'installation. La technologie de ces matériels est en évolution et ils devraient à terme être remplacés par des alimentations utilisant des techniques non irradiantes (magnétrons). Dans cette attente, il n'est pas prévu de classification temporaire des radars existants.

\section{Conclusion}

Bien que n'étant pas la finalité d'un radar, le mode de fonctionnement d'un tel dispositif entraine l'émission d'un rayonnement $\mathrm{X}$ parasite.

L'étude dosimétrique menée sur le radar Palmier, qui illustre parfaitement le rôle d'appui technique du SPRA en radioprotection au profit des forces, met en évidence l'exposition des personnels aux rayonnements ionisants à proximité des radars, risque méconnu et rarement publié dans la littérature scientifique (Degrave et al., 2009).

Cette étude dosimétrique montre que les niveaux d'exposition aux rayonnements $\mathrm{X}$ parasites des personnels affectés aux opérations de maintenance du radar Palmier restent faibles mais peuvent atteindre $1 \mathrm{mSv}$ par an au maximum.

Pendant une période probatoire d'un à trois ans, il a donc été décidé une classification des personnels comme travailleurs exposés de catégorie B et d'une surveillance dosimétrique individuelle externe passive et opérationnelle compte tenu du zonage adopté.

En fonction des niveaux observés, ces mesures de prévention devront être réévaluées à l'issue de cette période probatoire par le chef d'établissement, après avis de la PCR et du médecin de prévention.

Remerciements. Les auteurs remercient chaleureusement pour leur participation active à cette étude les équipes du bureau de sécurité radiologique du Service de protection radiologique des armées. 


\section{RÉFÉRENCES}

ASN (2008) Circulaire DTG/ASN nº 1 du 18 janvier 2008, relative à l'arrêté du 15/5/06 relatif aux conditions de délimitation et $\mathrm{d}$ signalisation des zones surveillées et contrôlées et des zones spécialement réglementées ou interdites, compte tenu de l'exposition aux rayonnements ionisants, ainsi qu'aux règles d'hygiène, de sécurité et d'entretien qui y sont apposées.

ASN (2010) Circulaire DGT/ASN n ${ }^{\circ} 4$ du 21 avril 2010 relative aux mesures de prévention des risques d'exposition aux rayonnements ionisants.

Degrave E., Meeusen B., Grivegnee A.R., et al. (2009) Causes of death among Belgian professionnal military radar operators: a 37-year retrospective cohort study, Int. J. Cancer 124 (4), 945-951.

IRSN (2010) Guide pratique Réalisation des études dosimétriques de poste de travail présentant un risque d'exposition aux rayonnements ionisants (version 2), DRPH/DIR n ${ }^{\circ} 2010-1$.

JORF (2006) Arrêté du 15 mai 2006 relatif aux conditions de délimitation et de signalisation des zones surveillées et contrôlées et des zones spécialement réglementées ou interdites compte tenu de l'exposition aux rayonnements ionisants, ainsi qu'aux règles d'hygiène, de sécurité et d'entretien qui y sont imposées, Journal Officiel de la République Française du 15 juin 2006.

JORF (2010) Arrêté du 21 mai 2010 portant homologation de la décision n 2010-DC-0175 de l'Autorité de sûreté nucléaire du 4 février 2010 précisant les modalités techniques et les périodicités des contrôles prévus aux articles R. 4452-12 et R. 4452-13 du code du travail ainsi qu'aux articles R. 1337-7 et R. 1333-95 du code de la santé publique. Journal Officiel de la République Française du 15 août 2010.

SPRA (2005) Arrêté du 10 janvier 2005 relatif aux attributions du service de protection radiologique des armées. 